\title{
Workflow Patterns as a Means to Model Task Succession in Games: A Preliminary Case Study
}

\author{
Simone Kriglstein ${ }^{1}$, Ross Brown ${ }^{2}$, and Günter Wallner ${ }^{3}$ \\ 1 Vienna University of Technology, Vienna, Austria \\ kriglstein@cvast.tuwien.ac.at \\ ${ }^{2}$ Queensland University of Technology, Brisbane, Australia \\ r.brown@qut.edu.au \\ 3 University of Applied Arts Vienna, Vienna, Austria \\ guenter.wallner@uni-ak.ac.at
}

\begin{abstract}
Over the last decade, people involved in game development have noted the need for more formal models and tools to support the design phase of games. In this paper we present an initial investigation into whether workflow patterns - which have already proven to be effective for modeling business processes - are a suitable way to model task succession in games. Our preliminary results suggest that workflow patterns show promise in this regard, but some limitations, especially with regard to time constraints, currently restrict their potential.
\end{abstract}

Keywords: Game Design, Design Tools, Workflow Patterns.

\section{Introduction}

Over the last decade, people involved in game development have repeatedly voiced the need for more formal models and tools for designing games. The traditional, and still used, game design document, a detailed and mostly textual description of all aspects of a game design (cf. 1]) has been criticized for several drawbacks. The design document is time-consuming to create, while at the same time being rarely read by members of the development team (see, e.g., [2]) and needs constant maintenance to keep the information up-to-date with highly iterative game development processes (cf. [2, 3]). Kreimeier [4], in a survey of game design methods, noted that the informal discussion of game design makes it difficult to put individual insight into a context of established knowledge. Therefore, formal visual languages have been proposed to more effectively express, abstract and communicate gameplay concepts or relationships between them (e.g., [2, 5,7$]$ ).

In a related context, Nelson and Mateas 8] point out that game development lacks tools to visualize and reason about systems of game mechanics on an abstract level. However, game designers agree that prototyping is essential for verifying design ideas (cf. [1, 9]). Yet, prototyping can be a time-consuming and costly process. On that account, researches have therefore proposed prototyping 
tools to visually model and simulate different aspects of game design on an abstract level (see, e.g., [3, 10, 11]).

In this paper we present an investigation into whether the widely supported workflow patterns [12] are a suitable way to model the succession of tasks players have to perform in a game. In a similar manner to Van Der Aalst et al. [12], we have sought to use a pattern analysis approach to identifying recurring structures within lists of activities performed by players in computer games. Such a pattern approach provides independence from the underlying game implementation environments, and the underlying theoretical languages used to describe such flows of activities. A workflow patterns approach enables us to specify abstractly the tasks to be performed by players within game environments, as the tasks given to players may share similarities in structure to tasks in business systems. The future benefits of such an approach are thus the ability to define such gameplay activity patterns in a rigorous and formal manner (drawing from the Petri net formalisms of workflow patterns) giving us both the power to deeply analyze their correct execution, and then execute them in Petri net based systems.

\section{Related Work}

To date a lot of work has been conducted to establish a common design vocabulary, for instance, in the form of design patterns used to express clearly intentions, analyses, and opinions regarding gameplay [13].

While design patterns are usually expressed in natural language, some game researchers have argued for more visual models to more clearly and compactly express and communicate ideas about game design (see the recent survey of Almeida and Silva [1] for a discussion on this subject). For example, Koster [5] presented a graphical notation system as a complement to classic game design documents to better communicate the game design. Bura [6], building upon the work of Koster [5], proposed a visual grammar which was influenced by Petri nets resembling data-flow representations of processes (whereas we are concerned with a control-flow perspective). Araújo and Roque [10] also applied Petri nets to model games and emphasized that the formal semantics of Petri nets can be leveraged to formally analyze and simulate game design elements already in an early design stage (e.g., to detect unwanted behavior).

Recently, Dormans [3] developed the Machinations tool, a graphical notation framework to express the rules of and simulate game economies in order to balance a game's economy or to prevent dominant strategies. While borrowing concepts from Petri nets, Machinations diagrams are aimed to be less complex and more accessible to designers. The work has been later extended by Klint and van Rozen [11] to allow not only for simulation but also for formal analysis of game designs. While the above mentioned works aim at expressing game mechanics, Cook [7] focused on the player experience and proposed skill chain diagrams to visually represent how players learn and acquire skills.

Workflow software technology has been applied to games by Brown et al. [14] who used a workflow language to script game tasks. 


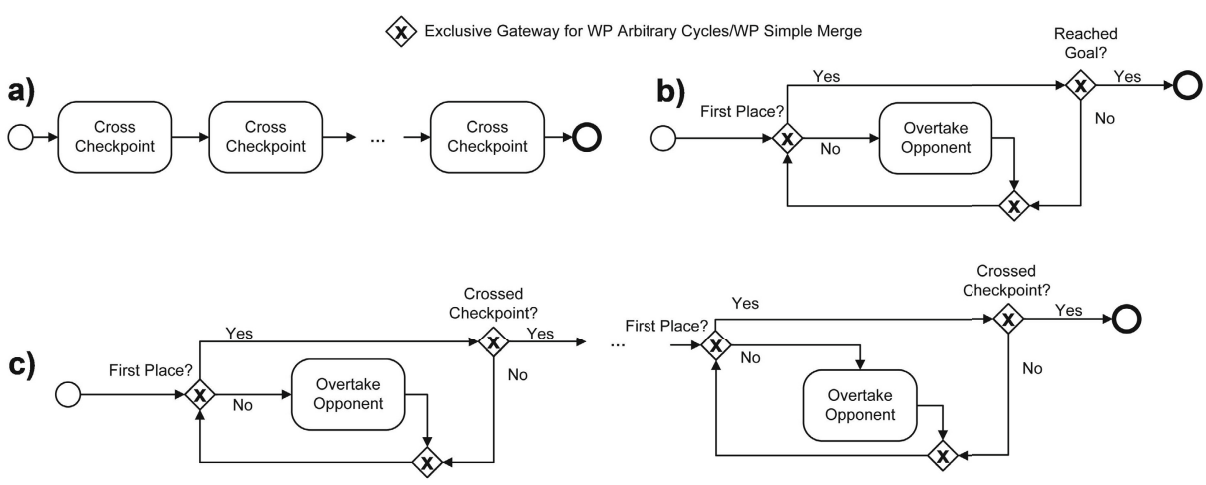

Fig. 1. The general three workflow patterns for the four main race events of NFS illustrated using the BPMN 2.0 visual grammar [15]: a) Pattern for checkpoint race event, b) pattern for sprint and for rival race event, and c) pattern for battle race event

\section{Use Cases}

For our initial investigation we have chosen two gameplay distinct action games, Need for Speed: The Run and Half Life 2.

Need For Speed: The Run (NFS): NFS is an action racer where the player takes part in a race across the US. The complete race is broken down into stages with each stage again divided into different race events. These events can roughly be divided into: a) checkpoint races, b) sprint races, c) rival races, and d) battle races. In case of a checkpoint race, a sequence of checkpoints has to be crossed. In workflow terms this can be described with a Sequence pattern (Workflow Pattern \#1 [17]). This is illustrated in Fig. 17.

A bit more complex is the workflow pattern for the sprint race event (see Fig. 10), where the player has to overtake a number of opponents and to cross the goal line in first position. The task to overtake multiple opponents can be described as a cycle that repeats until the player is on the first place (WP \#10 Arbitrary Cycles [17]). However, overtaking all opponents and reaching the first place is not enough to win the race, the player also has to maintain the lead until the goal line is reached. This is modeled with a second arbitrary cycle. The Simple Merge pattern (WP \#5 [17]) is used to merge the outgoing branch of the Overtake Opponent node and the false branch of the second arbitrary cycle.

The rival race event is technically the same as the sprint race event except that the player has to race against specific drivers that are part of the storyline. It can therefore be modeled the same way as the sprint race.

The battle race is a slight modification of the sprint race, where the player has to overtake an opponent and keep the lead until a checkpoint is reached after which the player races against another opponent until the next checkpoint. This repeats several times during a single battle race. The basic structure of the workflow pattern for a battle race event is therefore similar to the workflow 

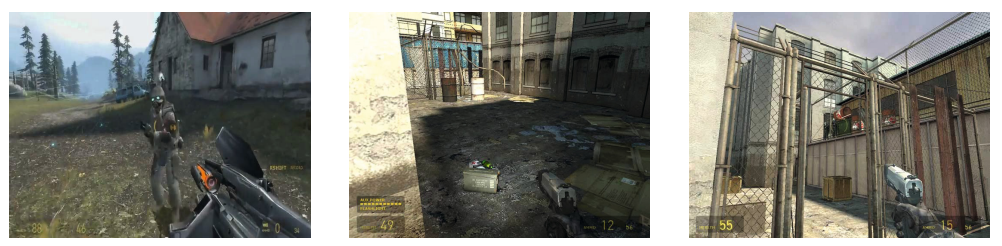

Fig. 2. Examples of the major tasks performed in an FPS - from left to right: Kill Agent, Use Resources (ammunition, healing items) and Open Door

pattern for a speed/rival race event with the difference that it repeats several times in sequential order (see Fig. 11).

Half Life 2 (HL2): HL2 is a single player first-person shooter (FPS). Like a number of first person shooters, you acquire weapons and health packs in order to defeat non-player characters (NPCs) and traverse levels. Analysis of the levels in the game indicate a number of workflow patterns. First of all, entrance to a level triggers instancing of gameplay tasks (WP \#6 - Multi-Choice [17] in combination with WP \#8 - Multi-Merge [17]). We group these into three major task types, viz. Kill Agents, Use Resources, Open Doors (see Fig. 21). Kill Agents is the destruction, by whatever means, of other agents in the game. Open Door tasks are a general description of having to remove a physical impediment to progress spatially, for example, opening a door, destroying an oil drum, or killing a blocking NPC. These sequences are embedded within a set of multiple tasks that may be ignored. For example, you do not have to kill all agents to progress through a level. This killing of agents and collection of resources is often up to the discretion of the player. A subset of the tasks require a certain sequence (WP \#1 [17]) for them to be completed.

In the level Routekanal1 there are certain sequences that must be performed before progression can occur. For example, you clear out an area of NPCs with a mounted machine gun (Kill Agents) and come to an oil drum that has to be destroyed (Open Door) before you can traverse to the next location and enter a tunnel. Note, it is possible to avoid shooting the NPCs if you are skilled enough in traversal, but you must shoot the oil drum. Therefore, this is an example of an enforced sequence within multi-choices. Once the level is traversed, a final door opening task is completed to exit the level; left over tasks are removed from the list allocated to the player. You cannot go back to the level to finish the other remaining tasks, you must recommence the level in order to begin again, or, you must go to a save point in the game to recommence the level at the same state. This is an example of the Canceling Discriminator pattern (WP \#29 [17]).

Combining these four workflow patterns together, we construct an overall FPS workflow pattern shown in Fig. 3 (left). We argue that this pattern, in slight variations, forms the structure of a number of FPS games. Thus designs for other FPS games can be configured from an executable form of such a pattern.

In addition, there is further detail in the tasks being performed in the cancellation region. In a similar manner, such tasks may be devolved into a split 

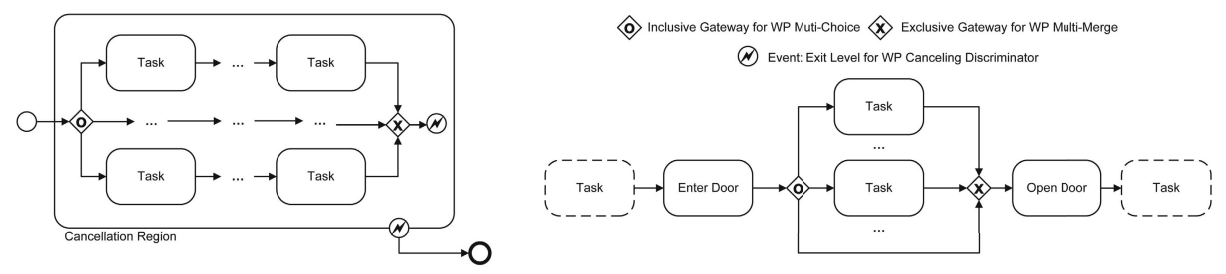

Fig. 3. Left: General FPS workflow pattern for HL2. Right: Exploded BPMN view of tasks in the left image, illustrating how the door opening tasks are key to enforcing particular orderings of tasks in HL2 as an FPS

upon opening a door and entering a region of a level. We define that a level is broken up into regions of gameplay, with door opening choke points, that enforce a sequence, but contain within them multi-choices and merging, as per Fig. 3 (right). Note that this pattern has no cancellation upon entering a new region. So the tasks in the split can be circumvented at the discretion of the player.

\section{Discussion}

In the presented use cases we focused on the description of the succession of tasks which players have to perform to successfully complete a level, but we did not consider implications of failure. However, modeling the consequences of failing a task can be equally important in game design (e.g., what happens if a checkpoint in a racing game is not reached). Workflow patterns may also be suitable for this task, for example, the WP \#4 - Exclusive Choice [17] can be used to describe branching depending on if the previous tasks were completed successfully or not.

Workflow patterns can be used to describe tasks on different levels of abstraction (e.g., opening a door could be described simply as Open Door or in more detail by modeling what actions are necessary to actually open it, for instance, Pull Lever followed by Turn Knob). Furthermore, a combination of the different abstraction levels can be used, for example, to provide a high-level overview with more detailed descriptions on demand.

One of the biggest limitations of the original control flow workflow patterns [12] for modeling task succession is perhaps their limited support of time aspects, e.g., time spans or timeouts. However, time aspects play a very important role in games. For example, in the above NFS use case the player has to reach the checkpoints within a predefined time limit. Future work can focus on analyzing how new time-based patterns (e.g., [16]) can be used to describe time critical game tasks.

\section{Conclusions}

In this paper we investigated the applicability of workflow patterns for modeling task succession in games. For this purpose we have chosen two different games as 
initial case studies. Our first results suggest that workflow patterns show some promise, but that there are also some limitations that currently constrain their potential in regard to games and which need further investigation, in particular, the ability to model winning and losing conditions and time aspects. Further case studies will be necessary to confirm our preliminary observations.

\section{References}

1. Almeida, M.S.O., da Silva, F.S.C.: A systematic review of game design methods and tools. In: Anacleto, J.C., Clua, E.W.G., da Silva, F.S.C., Fels, S., Yang, H.S. (eds.) ICEC 2013. LNCS, vol. 8215, pp. 17-29. Springer, Heidelberg (2013)

2. Librande, S.: One page designs. Presentation at the Game Developers Conference (2010)

3. Dormans, J.: Engineering Emergence: Applied Theory for Game Design. PhD thesis. Amsterdam University of Applied Sciences (2012)

4. Kreimeier, B.: Game design methods: A 2003 survey (2003), http://www.gamasutra.com/view/feature/2892/ game_design_methods_a_2003_survey.php (accessed: February 2014)

5. Koster, R.: A grammar of gameplay: Game atoms: can games be diagrammed? Presentation at the Game Developers Conference (2005)

6. Bura, S.: A game grammar (2006), http://www.stephanebura.com/diagrams/(accessed: February 2014)

7. Cook, D.: The chemistry of game design (2007), http://www.gamasutra.com/ view/feature/129948/the_chemistry_of_game_design.php (accessed: February 2014)

8. Nelson, M.J., Mateas, M.: A requirements analysis for videogame design support tools. In: Proc. of the 4th International Conference on Foundations of Digital Games. ACM (2009)

9. Neil, K.: Game design tools: Time to evaluate. In: Proc. of 2012 DiGRA Nordic. University of Tampere (2012)

10. Araújo, M., Roque, L.: Modeling games with petri nets. In: Atkins, B., Kennedy, H., Krzywinska, T. (eds.) Breaking New Ground: Innovation in Games, Play, Practice and Theory: Proc. of the 2009 DiGRA Conference. Brunel University (2009)

11. Klint, P., van Rozen, R.: Micro-machinations: A DSL for game economies. In: Erwig, M., Paige, R.F., Van Wyk, E. (eds.) SLE 2013. LNCS, vol. 8225, pp. 36-55. Springer, Heidelberg (2013)

12. van der Aalst, W.M.P., ter Hofstede, A.H.M., Kiepuszewski, B., Barros, A.P.: Workflow patterns. Distrib. Parallel Databases 14(1) (July 2003)

13. Björk, S.: Game design patterns 2.0, http://gdp2.tii.se (accessed: February 2014)

14. Brown, R., Lim, A., Wong, Y., Heng, S.M., Wallace, D.: Gameplay workflow: A distributed game control approach. In: Proc. of the 2006 International Conference on Game Research and Development. Murdoch University (2006)

15. Object Management Group: Business process model and notation version 2.0 (2011), http://www.omg.org/spec/BPMN/2.0/ (accessed: April 2014)

16. Niculae, C.: Time patterns in workflow management systems. Technical Report BPMcenter.org (2011)

17. Workflow Patterns Initiative: Control-flow patterns (2010), http://www.workflowpatterns.com/patterns/control/ (accessed: April 2014) 